\title{
Prospective evaluation of the capillaroscopic skin ulcer risk index in systemic sclerosis patients in clinical practice: a longitudinal, multicentre study
}

Ulrich A. Walker ${ }^{1 *+}$, Veronika K. Jaeger ${ }^{1 \dagger}$, Katharina M. Bruppacher ${ }^{2}$, Rucsandra Dobrota $^{3}$, Lionel Arlettaz ${ }^{4}$, Martin Banyai ${ }^{5}$, Jörg Beron ${ }^{6}$, Carlo Chizzolini ${ }^{7}$, Ernst Groechenig ${ }^{8}$, Rüdiger B. Mueller ${ }^{9}$, François Spertini ${ }^{10}$, Peter M. Villiger ${ }^{11}$ and Oliver Distler ${ }^{3}$

\begin{abstract}
Background: Nailfold capillaroscopy (NC) is an important tool for the diagnosis of systemic sclerosis (SSC). The capillaroscopic skin ulcer risk index (CSURI) was suggested to identify patients at risk of developing digital ulcers (DUs). This study aims to assess the reliability of the CSURI across assessors, the CSURI change during follow-up and the value of the CSURI in predicting new DUs.

Methods: This multicentre, longitudinal study included SSC patients with a history of DUs. NC images of all eight fingers were obtained at baseline and follow-up and were separately analysed by two trained assessors.

Results: Sixty-one patients were included (median observation time 1.0 year). In about $40 \%$ of patients (assessor 1 , $n=24,39 \%$; assessor $2, n=26,43 \%)$ no megacapillary was detected in any of the baseline or follow-up images; hence the CSURI could not be calculated.

In those 34 patients in whom CSURI scores were available from both assessors (26\% male; median age 57 years) the median baseline CSURI was 5.3 according to assessor 1 (IQR 2.6-16.3), increasing to 5.9 (IQR 1.3-12.0) at follow-up. According to assessor 2, the CSURI diminished from 6.4 (IQR 2.4-12.5) to 5.0 (IQR 1.7-10.0).

The ability of a CSURI $\geq 2.96$ category to predict new DUs was low (for both assessors, positive predictive value $38 \%$ and negative predictive value 50\%) and the inter-assessor agreements for CSURI categories were fair to moderate.
\end{abstract}

Conclusions: In this study, around $40 \%$ of patients could not be evaluated with the CSURI due to the absence of megacapillaries. Clinical decisions based on the CSURI should be made with caution.

Trial registration: Current Controlled Trials, ISRCTN04371709. Registered on 18 March 2011.

Keywords: Systemic sclerosis, Nailfold capillaroscopy, Capillaroscopic skin ulcer risk index, Inter-rater reliability, Digital ulcer prediction

\footnotetext{
* Correspondence: ulrich.walker@usb.ch

+Ulrich A Walker and Veronika K Jaeger contributed equally to this work.

${ }^{1}$ Department of Rheumatology, University Hospital Basel, Petersgraben 4,

4032 Basel, Switzerland

Full list of author information is available at the end of the article
}

(c) The Author(s). 2018 Open Access This article is distributed under the terms of the Creative Commons Attribution 4.0 International License (http://creativecommons.org/licenses/by/4.0/), which permits unrestricted use, distribution, and reproduction in any medium, provided you give appropriate credit to the original author(s) and the source, provide a link to the Creative Commons license, and indicate if changes were made. The Creative Commons Public Domain Dedication waiver (http://creativecommons.org/publicdomain/zero/1.0/) applies to the data made available in this article, unless otherwise stated. 


\section{Background}

Systemic sclerosis (SSc) is a chronic connective tissue disease characterised by endothelial cell dysfunction and fibrosis of the skin and internal organs $[1,2]$. Microangiopathy is one of the main histopathologic features detectable early in the course of the disease [3]. A gradual progression of vascular abnormalities has been observed during SSc progression [4]. Nailfold capillaroscopy (NC) is an imaging technique that detects morphological abnormalities of nailfold microcirculation. Furthermore, NC is an important tool for the classification and diagnosis of SSc in clinical practice $[5,6]$. The three NC patterns early, active and late were found to be associated with Raynaud's phenomenon (RP) as well as with the duration of the disease, possibly reflecting SSc evolution [4]. Although the diagnostic value of the NC patterns is well defined [7], different methodologies have been proposed to assess quantitative NC abnormalities in the follow-up of patients with SSc. However, their clinical applicability remains uncertain.

Sebastiani et al. [8] proposed the capillaroscopic skin ulcer risk index (CSURI) in 2009, as a quantitative measure of nailfold capillary damage that predicts the appearance of new digital ulcers (DUs) as well as the persistence of pre-existing DUs [8,9]. The CSURI is based on the number of capillaries in the distal nailfold capillary row and the number of megacapillaries, as well as the maximum diameter of the megacapillaries on capillaroscopic evaluation $[8,9]$.

In order to gain better insight into the value of monitoring quantitative $\mathrm{NC}$ abnormalities in clinical practice, this multicentre study was designed to describe the reliability of the CSURI across different trained assessors, to describe the change of CSURI during follow-up, to assess the value of the CSURI in predicting new DUs and to assess associations between the CSURI and demographic and disease characteristics.

\section{Methods}

\section{Study population and design}

This multicentre, prospective, observational study was carried out across eight sites in Switzerland between 2011 and 2015. Adult patients fulfilling the 1980 American College of Rheumatology criteria for SSc and with a history of DUs were included [10]. DUs were defined as a painful area with visually discernible depth and a loss of continuity of epithelial coverage which can be denuded or covered by a scab or necrotic tissue and is vascular in origin. Fissures, paronychia, extrusion of calcium or ulcers over the metacarpophalangeal joints or elbows are not regarded as DUs. In order to be included in this analysis, patients were also required to have at least one follow-up visit; if a patient had more than one follow-up visit, the last one was chosen as the follow-up visit. All inclusion and exclusion criteria are summarised in Additional file 1: Table S1.

This study was approved by the centres' ethic committees and each patient provided written informed consent.

Demographic patient characteristics and routine clinical data were recorded prospectively on a web-based electronic data capture system. Table 1 presents a description of the data collected. Patients underwent NC at baseline and at follow-up visits. Follow-up visits were performed if deemed necessary by the centres' physicians, but were recommended at 3, 6 and 12 months. Regular external monitoring with primary data verification was performed to ensure data quality.

Prior to commencing the study, the study sites' investigators were trained at an investigator meeting to perform NC. The nailfolds of eight fingers (digits 2-5 on both hands) were examined using the same NC device equipped with a $200 \times$ lens with LED illumination and an immersion fluid contact adapter (Optilia instruments $\mathrm{AB}$, Sollentuna, Sweden) in all centres. Four images

Table 1 Description of collected data

\begin{tabular}{|c|c|}
\hline & Demographics \\
\hline & Age (years) \\
\hline & Sex (female/male) \\
\hline & Smoking habit (never smoker/ex-smoker/current smoker) \\
\hline & Disease characteristics \\
\hline & Time since RP onset (years) \\
\hline & Time since first non-RP manifestation (years) \\
\hline & Cutaneous involvement (limited/diffuse) \\
\hline & Modified Rodnan skin score (range 0-51) \\
\hline & $\begin{array}{l}\text { Erectile dysfunction (yes/no; defined as a score below } 22 \text { in the } \\
\text { International Index for Erectile Dysfunction-5 [19]) }\end{array}$ \\
\hline & Kidney involvement (yes/no; defined as proteinuria) \\
\hline & History of renal crisis (yes/no) \\
\hline & RP condition score (range $0-10$ ) \\
\hline & $\begin{array}{l}\text { DUs (yes/no; defined as a painful area with visually discernible depth } \\
\text { and a loss of continuity of epithelial coverage, which can be denuded } \\
\text { or covered by a scab or necrotic tissue and is vascular in origin; DUs } \\
\text { do not include fissures, paronychia, extrusion of calcium or ulcers } \\
\text { over the metacarpophalangeal joints or elbows.) }\end{array}$ \\
\hline & Time since first DU (years) \\
\hline & Number of DUs \\
\hline & $\begin{array}{l}\text { Major digital vascular complications (none/soft tissue infection/ } \\
\text { gangrene/autoamputation) }\end{array}$ \\
\hline & $\begin{array}{l}\text { Laboratory (measured according to local standards in the } \\
\text { respective centres) }\end{array}$ \\
\hline & Antinuclear autoantibody positivity (yes/no) \\
\hline & Anticentromere autoantibody positivity (yes/no) \\
\hline & Anti-topoisomerase autoantibody positivity (yes/no) \\
\hline
\end{tabular}

DU digital ulcer, RP Raynaud's phenomenon 
across the nailfold quadrants of each finger were obtained. Digital NC images were stored centrally and examined separately at the end of the study by two identically trained central assessors (UAW and OD). The central assessors were blinded for the patients, the temporal sequence of the fingers and the scoring results of the other assessor. In each NC image, the assessors assessed the total number of capillaries in the distal row, the number of megacapillaries and the maximum diameter of the megacapillaries. Additionally, the images were also evaluated locally at the centres (local assessors). The qualitative assessment-that is, the NC pattern (early/active/late) was performed by one additional central assessor (RD).

The presence of at least one megacapillary is necessary to calculate the CSURI $[8,9]$. The CSURI is calculated for only one image per patient per time point; this image is identified based on the lowest number of capillaries in the distal row as the first criterion and subsequently the highest number of megacapillaries as the second criterion $[8,9]$. As described in detail elsewhere, the number of megacapillaries is multiplied by the maximum diameter of the megacapillaries and then divided by the square of the number of capillaries to form the CSURI $[8,9]$. For part of the analysis, we categorised the CSURI at 2.96, a threshold which was suggested to be predictive for the prospective development of DUs [9].

\section{Data analysis}

Categorical variables were calculated as frequencies and percentages, and continuous variables were calculated as means with standard deviation (SD) or medians with interquartile range (IQR). Chi-square tests/Fisher's exact tests and Mann-Whitney $U$ tests were applied for across-group comparisons. Intraclass correlation coefficients and Cohen's $\mathrm{K}$ were calculated to assess the agreement between the two assessors. Linear regression analysis was applied to evaluate associations between the change in CSURI between baseline and follow-up and demographic or disease characteristics. All statistical analyses were performed with Stata/IC 13.1 (StataCorp., College Station, TX, USA).

\section{Results}

Between 2011 and 2015, 61 patients from eight centres were enrolled. The median observation time was 1.0 year
(IQR 1.0-1.1). Of these 61 patients, 24 patients according to central assessor $1(39 \%)$ and 26 patients according to central assessor 2 (43\%) had no megacapillaries present on any assessed finger either at baseline or at the follow-up visit (Table 2). Due to the absence of megacapillaries, the CSURI could not be calculated for those patients. Therefore, for only 34 of the 61 eligible patients (56\%) was the CSURI scorable by both central assessors at both time points. This percentage of patients without megacapillaries was similar across all eight centres $(p=0.72)$.

According to both central assessors, megacapillaries were present in 43 patients at baseline (Table 2); 30\% of these showed an early SSc pattern on NC, $44 \%$ an active pattern and $26 \%$ a late pattern. Of the 18 patients without megacapillaries present at baseline (Table 2), 6\% (one patient) had an early pattern, $28 \%$ a late pattern and the remaining $66 \%$ of patients showed no SSc specific pattern on $\mathrm{NC}$ at baseline.

The following analyses are entirely based on the $34 \mathrm{pa}-$ tients with an available CSURI by both central assessors at both time points, named the study population.

The baseline characteristics of the study population are presented in Table 3. The median observation time in this population was also 1.0 year (IQR 1.0-1.1). There were no statistically significant differences between the patients included in the study population and those excluded from further analysis. The included patients were, however, slightly younger (median age 57 years vs 62 years) and nominally more often had diffuse skin involvement $(41 \%$ vs $33 \%)$ than the excluded patients. As many as $24 \%$ of the patients had experienced ulcer complications (soft tissue infections and gangrene).

In the study population, central assessor 1 counted a median of five capillaries in the distal row (range 2-10) and a median of one megacapillary (range 1-6) with a median maximum diameter of $62.5 \mu \mathrm{m}$ (range 50-130 $\mu \mathrm{m})$. Central assessor 2 counted a median of five capillaries in the distal row (range 2-10) and two megacapillaries (range 1-20) with a median diameter of $75 \mu \mathrm{m}$ (range $30-180 \mu \mathrm{m}$ ).

The median baseline CSURI scores were 5.3 (IQR 2.616.3) as evaluated by central assessor 1 and 6.4 (IQR 2.4-12.5) as evaluated by central assessor 2 . The median

Table 2 Overview of distribution of patients with absent megacapillaries at any of the assessed fingers (i.e. CSURI non-scorability) at baseline and follow-up according to the central assessors

\begin{tabular}{llll}
\hline & \multicolumn{3}{l}{ SSc patients (out of 61 patients) who had no megacapillaries on any of the assessed fingers } \\
\cline { 2 - 4 } & Central assessor 1 & Central assessor 2 & Both central assessors combined \\
\hline Baseline & 15 patients (25\%) & 17 patients (28\%) & 18 patients (30\%) \\
Follow-up & 15 patients (25\%) & 18 patients (30\%) & 18 patients (30\%) \\
Any of the two time points & 24 patients (39\%) & 26 patients (43\%) & 27 patients (44\%) \\
\hline
\end{tabular}

CSURI capillaroscopic skin ulcer risk index, SSC systemic sclerosis 
Table 3 Comparison of baseline demographics and disease characteristics between patients included in this analysis (scorable CSURI at baseline and follow-up) and those excluded (CSURI not scorable at baseline and follow-up)

\begin{tabular}{|c|c|c|c|}
\hline Baseline characteristic of study population & Included & Excluded & $p$ value \\
\hline$N$ & 34 & 27 & \\
\hline Age (years) & $56.6(47.8-64.8)$ & $61.7(53.6-64.6)$ & 0.25 \\
\hline Male sex & 26 & 30 & 0.79 \\
\hline \multicolumn{4}{|l|}{ Smoking habit } \\
\hline Never smoker & 47 & 37 & \multirow[t]{3}{*}{0.23} \\
\hline Ex-smoker & 18 & 37 & \\
\hline Current smoker & 35 & 26 & \\
\hline Bosentan at any time during the observation period & 38 & 33 & 0.69 \\
\hline \multicolumn{4}{|l|}{ Disease characteristics } \\
\hline Time since RP onset (years) & $7.0(3-15)$ & $5.0(2-21)$ & 0.65 \\
\hline Time since first non-RP manifestation (years) & $4.5(1-9)$ & $5.0(2-12)$ & 0.44 \\
\hline \multicolumn{4}{|l|}{ Cutaneous involvement } \\
\hline Limited & 59 & 67 & \multirow[t]{2}{*}{0.53} \\
\hline Diffuse & 41 & 33 & \\
\hline Erectile dysfunction & 13 & 44 & 0.29 \\
\hline Kidney involvement & 0 & 4 & 0.45 \\
\hline History of renal crisis & 0 & 0 & - \\
\hline RP condition score [20] & $3.8(2-7)$ & $5.0(2-7)$ & 0.49 \\
\hline mRSS & $8(6-13)$ & $9(4-18)$ & 0.63 \\
\hline Time since first DU (years) & $1.5(0.7-4.2)$ & $2.3(1.1-5.0)$ & 0.42 \\
\hline DU & 76 & 74 & 0.83 \\
\hline Number of DUs (in patients with DUs) & $3.0(1-7)$ & $3.5(1-6)$ & 0.99 \\
\hline \multicolumn{4}{|l|}{ Previous major digital vascular complication } \\
\hline None & 76 & 69 & \multirow[t]{4}{*}{0.39} \\
\hline Soft tissue infection & 21 & 15 & \\
\hline Gangrene & 3 & 8 & \\
\hline Autoamputation & 0 & 8 & \\
\hline \multicolumn{4}{|l|}{ Laboratory parameters } \\
\hline ANA positive & 100 & 96 & 0.25 \\
\hline ACA positive & 48 & 45 & 0.83 \\
\hline Scl-70 positive & 34 & 45 & 0.46 \\
\hline
\end{tabular}

Data presented as \% or median (interquartile range)

ACA anticentromere autoantibodies, ANA anti-nuclear autoantibodies, CSURI capillaroscopic skin ulcer risk index, DU digital ulcer, mRSS modified Rodnan skin score, RP Raynaud's phenomenon, SCl-70 anti-topoisomerase I autoantibodies

baseline CSURI was 8.2 (IQR 4.5-23.6) according to the local assessors. According to central assessor 1, the median CSURI score increased to 5.9 (IQR 1.3-12.0) at follow-up, whereas the median CSURI as evaluated by central assessor 2 decreased to 5.0 (IQR 1.7-10.0) at follow-up. The correlation coefficient between the baseline CSURI of the two assessors was 0.42 , indicating a fair agreement [11]. There was a poor to fair agreement between the CSURI scored by the central assessors and the local assessors (central assessor 1/local assessors 0.45 ; central assessor $2 /$ local assessors 0.38 ).
As evaluated by central assessor 1, 35\% of patients had a higher CSURI at follow-up compared to $44 \%$ when evaluated by central assessor 2 . In only $40 \%$ of the 34 patients was the change in CSURI between baseline and follow-up in the same direction for both central assessors; that is, an increase as measured by both assessors, a decrease in the measurements of both assessors or no change (Fig. 1).

According to both central assessors, 10 patients (29\%) were in the low-risk category (CSURI $<2.96$ [9]) at baseline; however, only seven of those 10 patients were 


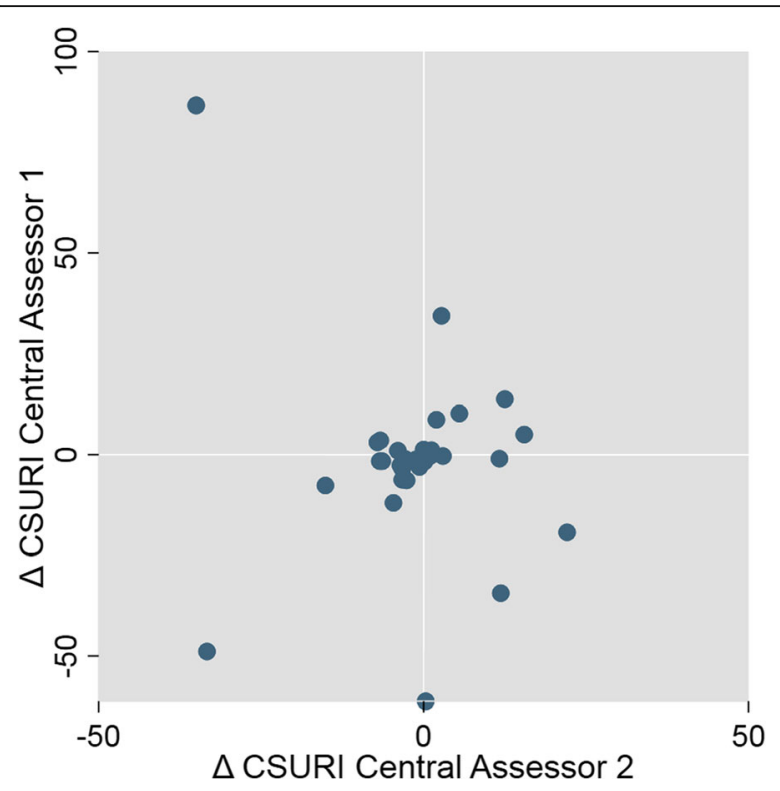

Fig. 1 Change in CSURI between baseline and follow-up as evaluated by central assessors 1 and 2. CSURI capillaroscopic skin ulcer risk index

concomitantly rated by both assessors as being in the low-risk category. According to the local assessors, five patients $(15 \%)$ were in the low-risk category; however, only two of those were concomitantly rated by both central assessors into the low-risk category. The inter-assessor agreement of the central assessors for the baseline CSURI risk category was 0.58 , indicating a moderate level of agreement [12]. The inter-assessor agreements of the local assessor and central assessor 1 or 2 were both 0.25 , indicating a fair agreement.

Central assessor 1 scored $88 \%$ of the patients into the same risk category at baseline and at follow-up (i.e. either low-low risk or high-high risk); the remaining $12 \%$ were in the low-risk category at follow-up, but in the high-risk category at baseline. According to assessor 2, $73 \%$ of patients were in the same risk category at baseline and follow-up, $21 \%$ were in the high-risk category at baseline and in the low-risk category at follow-up, and $6 \%$ were in the low-risk category at baseline and in the high-risk category at follow-up. The agreement between the two central assessors of this 'change in risk categories' was fair $(\kappa=0.37)$ [12]. There was no agreement between central assessor 1 and central assessor 2 and the local assessors regarding this 'change of risk category' ( $\kappa=-0.09, \kappa=-0.16$, respectively).

The prevalence of DU at baseline was 76\% (Table 3) compared to $59 \%$ at follow-up. The ability of CSURI $\geq$ 2.96 (i.e. the high-risk category) to predict a higher number of DUs at follow-up than at baseline visit was rather low (positive predictive value for both central assessors $38 \%$, for local assessors $48 \%$ ), as was the ability of CSURI
$<2.96$ (i.e. low-risk category) to predict fewer or the same number of DUs at follow-up compared to baseline (negative predictive value for both central assessors 50\%, for local assessors 67\%). Out of the 34 included patients, 28 patients were classified into the same risk category by both central assessors. The positive and the negative predictive values based on these 28 patients were similarly lower (positive predictive value $38 \%$, negative predictive value $43 \%$ ) than the predictive values based on all 34 patients. The predictive values in patients who were treated with bosentan at any time during the observation period were similar to those who were not treated with bosentan.

No demographic or disease characteristic was associated with the change in the CSURI between baseline and follow-up simultaneously for both CSURIs, the one scored by assessor 1 and the one scored by assessor 2, in univariate linear regression (Table 4).

\section{Discussion}

This prospective, longitudinal study examined the use of the CSURI in everyday clinical practice and demonstrates that $40 \%$ of patients in this multicentre study could not be evaluated with the CSURI at baseline and follow-up visits, mainly due to a normal NC pattern and the lack of any megacapillary as a prerequisite for the calculation of the CSURI $[9,13]$. Additionally, the agreement of the CSURI between the two trained and experienced assessors was mediocre at best, as was the agreement between the two central assessors and the local assessors.

Our high percentage of non-scorable patients contrasts with the first CSURI study and the CSURI validation study $[8,9]$. In the first study all patients had megacapillaries present, and in the second study only 13 out of an unselected SSc population of 242 patients (5\%) were excluded from the study due to the absence of megacapillaries $[8,9]$. However, in various other studies that were not applying the CSURI, the percentage of patients without megacapillaries was comparable to our high percentage. For instance, in a study of 188 SSc patients at least one quarter of patients had no megacapillaries [14]. Similarly, in two other studies, $24 \%$ and $30 \%$ of patients had no megacapillaries in any of the assessed fingers $[15,16]$. Our discrepancies with the first CSURI studies are difficult to explain with differences of equipment, given the fact that very similar devices were in use in the first CSURI studies as well as in our study. Our patient population had similar disease duration as the patients consecutively recruited into the first CSURI study [8], but a higher proportion of diffuse SSc patients (41\% vs $9 \%$ ), which may not explain the lower prevalence of megacapillaries in our study. 
Table 4 Univariate linear regression of $\Delta$ CSURI (defined as the difference of CSURI between baseline and follow-up) and demographics and disease characteristics $(n=34)$

\begin{tabular}{|c|c|c|c|c|c|c|}
\hline \multirow[t]{2}{*}{ Characteristic of study population } & \multicolumn{3}{|c|}{ Central assessor 1} & \multicolumn{3}{|c|}{ Central assessor 2} \\
\hline & $\bar{\beta}$ & $95 \% \mathrm{Cl}$ & $\overline{p \text { value }}$ & $\beta$ & $95 \% \mathrm{Cl}$ & $p$ value \\
\hline Age (years) & -0.095 & -0.75 to 0.56 & 0.77 & 0.222 & -0.08 to 0.53 & 0.15 \\
\hline Male sex & -0.92 & -19.2 to 17.4 & 0.92 & -3.5 & -12.2 to 5.3 & 0.42 \\
\hline \multicolumn{7}{|l|}{ Smoking habit } \\
\hline Never smoker & Reference & & & Reference & & \\
\hline Ex-smoker & 3.61 & -18.7 to 25.9 & 0.74 & -1.4 & -12.3 to 9.5 & 0.79 \\
\hline Current smoker & 11.54 & -6.2 to 29.3 & 0.20 & -3.9 & -12.7 to 4.8 & 0.36 \\
\hline \multicolumn{7}{|l|}{ Disease characteristics } \\
\hline Time since RP onset (years) & -0.093 & -0.75 to 0.56 & 0.77 & 0.096 & -0.19 to 0.39 & 0.50 \\
\hline Time since first non-RP manifestation (years) & -1.16 & -2.9 to 0.6 & 0.18 & 0.91 & 0.1 to 1.7 & 0.025 \\
\hline Time since first DU (years) & -0.81 & -3.0 to 1.4 & 0.46 & 0.90 & 0.0 to 1.8 & 0.046 \\
\hline \multicolumn{7}{|l|}{ Previous major digital vascular complication } \\
\hline None & Reference & & & Reference & & \\
\hline Soft tissue infection & -5.62 & -25.9 to 14.6 & 0.58 & 3.15 & -6.4 to 12.7 & 0.51 \\
\hline Gangrene & 5.24 & -43.2 to 53.7 & 0.83 & 14.74 & -8.1 to 37.5 & 0.20 \\
\hline \multicolumn{7}{|l|}{ Cutaneous involvement } \\
\hline Limited & Reference & & & Reference & & \\
\hline Diffuse & 4.05 & -12.3 to 20.4 & 0.62 & -3.08 & -10.9 to 4.8 & 0.43 \\
\hline Erectile dysfunction & -7.99 & -14.2 to -1.8 & 0.019 & -7.28 & -21.1 to 6.5 & 0.25 \\
\hline RP condition score [20] at baseline & -0.51 & -2.4 to 1.4 & 0.59 & -0.11 & -1.0 to 0.8 & 0.81 \\
\hline mRSS at baseline & -0.04 & -0.9 to 0.9 & 0.93 & -0.002 & -0.4 to 0.4 & 0.99 \\
\hline Number of DUs at baseline & 1.05 & -0.8 to 2.9 & 0.25 & 0.55 & -0.3 to 1.4 & 0.21 \\
\hline \multicolumn{7}{|l|}{ Laboratory parameters } \\
\hline ACA positive & 6.08 & -10.4 to 22.5 & 0.46 & -0.02 & -8.1 to 8.0 & 0.99 \\
\hline Scl-70 positive & -16.5 & -34.8 to 1.8 & 0.075 & -2.73 & -12.1 to 6.7 & 0.56 \\
\hline
\end{tabular}

ACA anticentromere autoantibodies, Cl confidence interval, CSURI capillaroscopic skin ulcer risk index, DU digital ulcer, mRSS modified Rodnan skin score, RP Raynaud's phenomenon, $\mathrm{SCl}-70$ anti-topoisomerase autoantibodies

In our study, the CSURI had only fair to moderate inter-rater reliability. This contrasts with an 'almost perfect' inter-observer reproducibility reported by Sebastiani et al. [8] in the original CSURI study, with $\mathrm{k}=0.96$ based on the CSURI, dichotomised at the 2.96 cut-off value. A slightly lower but still 'almost perfect' inter-rater agreement of 0.85 was found in the validation study [9]. It is unlikely that these discrepancies can be completely explained with a lack of experience or different training, as both central assessors were trained together by authors of the original CSURI publications and used the same digital images and imaging software.

The CSURI was created as a prognostic index to predict the onset of new DUs [8]. In a validation study, Sebastiani et al. [9] demonstrated high predictive values for the development of DUs within 3 months, especially a high negative predictive value of $97 \%$, but also a high positive predictive value of the CSURI of $81 \%$ in patients with a history of DUs. However, in another study by
Sebastiani et al. [17] a poorer performance of the CSURI with lower predictive values was also observed in a population of SSc patients treated with bosentan. Differences in DU prediction may therefore be explained by differences in vasoactive medications. When we stratified our patients by bosentan treatment, we did not observe major differences in predictive values. It must, however, also be kept in mind that the predictive values from our study should not be directly compared with the studies by Sebastiani et al. as we assessed the predictive values of a higher number of DUs at follow-up compared to baseline and not 'incident DU' as Sebastiani et al. Additionally, the time between the baseline and the follow-up visit was considerably longer in our study (median time 1 year) than in Sebastiani et al.'s studies (3 months), which could also partly explain the differences in the predictive power of the CSURI.

A recent systematic literature review critically appraising studies reporting the prognostic value of $\mathrm{NC}$ in SSc 
also assessed the predictive value of the CSURI [18]. In line with our study, Paxton and Pauling [18] conclude that it is difficult to draw robust conclusions regarding the prognostic role of the CSURI; the reason for this being high levels of potential biases relating to study confounding as well as the statistical analyses.

It needs to be mentioned that our study has a rather limited sample size, which restricts the power to assess CSURI predictors in terms of demographic and disease characteristics. However, the mediocre performance of the CSURI regarding the inter-rater differences, as well as the high number of patients who could not be included due to the absence of megacapillaries, will not be a result of chance alone, even if a larger sample size would naturally have been beneficial.

\section{Conclusions}

The CSURI was not applicable in a large percentage of patients due to the absence of megacapillaries and demonstrated only fair to moderate inter-rater reliability. Thus, in routine clinical practice, the CSURI should be used with caution for treatment decisions and prediction of incident DUs.

\section{Additional file}

Additional file 1: Table S1. Inclusion and exclusion criteria. (DOCX $16 \mathrm{~kb}$ )

\section{Abbreviations}

CSURI: Capillaroscopic skin ulcer risk index; DU: Digital ulcer; IQR: Interquartile range; NC: Nailfold capillaroscopy; RP: Raynaud's phenomenon; SD: Standard deviation; SSc: Systemic sclerosis

\section{Funding}

This study was sponsored by Actelion Pharma Schweiz AG. The company contributed to the design of the study and was involved in the interpretation of the data and the writing, review and approval of the publication.

\section{Availability of data and materials}

The datasets analysed during the current study are available from the corresponding author on reasonable request.

\section{Authors' contributions}

UAW, KMB and OD contributed to conception and design. UAW, RD, LA, MB, CC, EG, RBM, FS, PMV and OD contributed to acquisition of data. UAW, VKJ, $\mathrm{RD}, \mathrm{JB}$ and $\mathrm{OD}$ analysed and interpreted data. UAW, VKJ, RD, JB and OD drafted the article. All authors critically revised the manuscript for important intellectual content. All authors read and approved the final manuscript.

\section{Ethics approval and consent to participate}

Ethics approval was obtained from the following ethics committees: Ethikkommission Nordwest- und Zentralschweiz, Kantonale Ethikkommission Kanton Aargau, Commission Cantonale d'Éthique de la Recherche Genève, Commission cantonal (VD) d'éthique de la recherché sur l'être humain, Kantonale Ethikkommission Bern, Ethikkommission Ostschweiz and Kantonale Ethikkommission Zürich.

\section{Consent for publication}

Not applicable.

\section{Competing interests}

VKJ received travel grant and travel support from Actelion Pharma Schweiz AG. KMB was a former full-time employee of Actelion Pharma Schweiz AG. $\mathrm{RD}$ received research funding through an Articulum Fellowship sponsored by Pfizer (2013-2014), a EULAR training bursary and from the FP-7Desscipher project, and speaker fees from Actelion. JB is a full-time employee of Actelion Pharma Schweiz AG. CC received travel support and speaker fees from Actelion and Boehringer Ingelheim. OD has a consultancy relationship and/or research funding from AnaMar, Bayer, Boehringer Ingelheim, Catenion, CSL Behring, ChemomAb, Roche, GSK, Inventiva, Italfarmaco, Lilly, medac, Medscape, Mitsubishi Tanabe Pharma, MSD, Novartis, Pfizer, Sanofi and UCB in the area of potential treatments of scleroderma and its complications; in addition, OD has licensed patent mir-29 for the treatment of systemic sclerosis. The remaining authors declare that they have no competing interests.

\section{Publisher's Note}

Springer Nature remains neutral with regard to jurisdictional claims in published maps and institutional affiliations.

\section{Author details}

${ }^{1}$ Department of Rheumatology, University Hospital Basel, Petersgraben 4 4032 Basel, Switzerland. ${ }^{2}$ Bellikon, Switzerland. ${ }^{3}$ Department of Rheumatology, University Hospital Zurich, Zurich, Switzerland. ${ }^{4}$ Institut Central—Hôpital du Valais, Sion, Switzerland. ${ }^{5}$ Kantonsspital Luzern, Luzern, Switzerland. ${ }^{6}$ Actelion Pharma Schweiz AG, Baden, Switzerland. ${ }^{7}$ Immunology \& Allergy, University Hospital and School of Medicine, Geneva, Switzerland. ${ }^{8}$ Kantonsspital Aarau, Aarau, Switzerland. ${ }^{9}$ Kantonsspital St. Gallen, St. Gallen, Switzerland. ${ }^{10}$ Division of Immunology and Allergy, Centre Hospitalier Universitaire Vaudois, Lausanne, Switzerland. ${ }^{11}$ Department of Rheumatology, Immunology and Allergology, University Hospital and University of Bern, Bern, Switzerland.

Received: 23 July 2018 Accepted: 25 September 2018

Published online: 25 October 2018

\section{References}

1. Gabrielli A, Avvedimento EVEV, Krieg T. Scleroderma. N Engl J Med. 2009; 360(19):1989-2003.

2. Varga J, Trojanowska M, Kuwana M. Pathogenesis of systemic sclerosis: recent insights of molecular and cellular mechanisms and therapeutic opportunities. J Scleroderma Relat Disord. 2017;2(3):137-52.

3. Cutolo M, Sulli A, Smith V. Assessing microvascular changes in systemic sclerosis diagnosis and management. Nat Rev Rheumatol. 2010;6(10):57887.

4. Cutolo M, Pizzorni C, Secchi ME, Sulli A. Capillaroscopy. Best Pract Res Clin Rheumatol. 2008;22(6):1093-108.

5. van den Hoogen F, Khanna D, Fransen J, Johnson SR, Baron M, Tyndall A, et al. 2013 classification criteria for systemic sclerosis: an American College of Rheumatology/European League against Rheumatism collaborative initiative. Arthritis Rheum. 2013;65(11):2737-47.

6. Smith $V$, Thevissen $K$, Trombetta AC, Pizzorni C, Ruaro B, Piette $Y$, et al. Nailfold capillaroscopy and clinical applications in systemic sclerosis. Microcirculation. 2016;23(5):364-72.

7. Cutolo M, Smith V. Nailfold capillaroscopy. In: Scleroderma. Boston: Springer US; 2012. p. 331-46.

8. Sebastiani M, Manfredi A, Colaci M, D'amico R, Malagoli V, Giuggioli D, et al. Capillaroscopic skin ulcer risk index: a new prognostic tool for digital skin ulcer development in systemic sclerosis patients. Arthritis Rheum. 2009; 61(5):688-94.

9. Sebastiani M, Manfredi A, Vukatana G, Moscatelli S, Riato L, Bocci M, et al. Predictive role of capillaroscopic skin ulcer risk index in systemic sclerosis: a multicentre validation study. Ann Rheum Dis. 2012;71(1):67-70.

10. Masi AT, Rodnan GP, Medsger TA Jr, Altman RD, D'Angelo WA, Fries JF, et al Preliminary criteria for the classification of systemic sclerosis (scleroderma). Subcommittee for scleroderma criteria of the American Rheumatism Association Diagnostic and Therapeutic Criteria Committee. Arthritis Rheum. 1980:23(5):581-90.

11. Cicchetti DV, D V. Guidelines, criteria, and rules of thumb for evaluating normed and standardized assessment instruments in psychology. Psychol Assess. 1994;6(4):284-90. 
12. Landis JR, Koch GG. The measurement of observer agreement for categorical data. Biometrics. 1977;33(1):159-74.

13. Sebastiani M, Manfredi A, Cassone G, Giuggioli D, Ghizzoni C, Ferri C. Measuring microangiopathy abnormalities in systemic sclerosis patients: the role of capillaroscopy-based scoring models. Am J Med Sci. 2014;348(4): 331-6.

14. Pavan TR, Bredemeier M, Hax V, Capobianco KG, da Silva Mendonça Chakr R, Xavier RM. Capillary loss on nailfold capillary microscopy is associated with mortality in systemic sclerosis. Clin Rheumatol. 2018;37(2):475-81.

15. Ruaro B, Sulli A, Alessandri E, Pizzorni C, Ferrari G, Cutolo M, et al. Laser speckle contrast analysis: a new method to evaluate peripheral blood perfusion in systemic sclerosis patients. Ann Rheum Dis. 2014;73(6):1181-5.

16. Lüders S, Friedrich S, Ohrndorf S, Glimm A-M, Burmester G-R, Riemekasten $G$, et al. Detection of severe digital vasculopathy in systemic sclerosis by colour Doppler sonography is associated with digital ulcers. Rheumatology. 2017;56(11):1865-73.

17. Sebastiani M, Cestelli V, Manfredi A, Praino E, Cannarile F, Spinella A, et al. SAT0621 Validation Study of Predictive Value of Capillaroscopic Skin Ulcer Risk Index (CSURI) in Scleroderma Patients Treated with Bosentan. Ann Rheum Dis. 2015;74(Suppl 2):886.1-886.

18. Paxton D, Pauling JD. Does nailfold capillaroscopy help predict future outcomes in systemic sclerosis? A systematic literature review. Semin Arthritis Rheum. 2018. https://doi.org/10.1016/.jsemarthrit.2018.02.005.

19. Rosen RC, Cappelleri JC, Smith MD, Lipsky J, Peña BM. Development and evaluation of an abridged, 5 -item version of the International Index of Erectile Function (IIEF-5) as a diagnostic tool for erectile dysfunction. Int J Impot Res. 1999;11(6):319-26.

20. Merkel PA, Herlyn K, Martin RW, Anderson JJ, Mayes MD, Bell P, et al. Measuring disease activity and functional status in patients with scleroderma and Raynaud's phenomenon. Arthritis Rheum. 2002;46(9):2410-20.

Ready to submit your research? Choose BMC and benefit from:

- fast, convenient online submission

- thorough peer review by experienced researchers in your field

- rapid publication on acceptance

- support for research data, including large and complex data types

- gold Open Access which fosters wider collaboration and increased citations

- maximum visibility for your research: over $100 \mathrm{M}$ website views per year

At $\mathrm{BMC}$, research is always in progress.

Learn more biomedcentral.com/submissions 